\title{
Evaluation of dyspnea severity and sleep quality in patients with novel coronavirus
}

\author{
SERAP Güngör ${ }^{1}$, Betul Tosun ${ }^{2}$, Nursemin Unal ${ }^{3}$, and Ismail Dusak ${ }^{4}$ \\ ${ }^{1}$ Affiliation not available \\ ${ }^{2}$ Hasan Kalyoncu University \\ ${ }^{3}$ Ankara Medipol University \\ ${ }^{4}$ Sanliurfa Mehmet Akif Inan Training and Research Hospital
}

December 27, 2020

\begin{abstract}
Aim: Dyspnea, a common symptom of novel coronavirus, can negatively affect sleep quality. The aim of this study was to evaluate the relationship between dyspnea severity and sleep quality in patients with COVID-19. Study Design: A crosssectional design was used. Methods: Using the researcher's mobile phone, data were collected via an online questionnaire from patients $(n=100)$ who agreed to participate in the study. The data-collection form comprised three parts: a patient descriptive information form, the Dyspnea-12 Questionnaire, and the Richards-Campbell Sleep Questionnaire. Results: The mean age of patients was $46.39 \pm 12.61$ years and $66.0 \%$ were male. Patients who were treated in the intensive care unit, had bachelor's degree or more and patients with comorbid diseases had low mean scores from the RCSQ and high mean scores from the Dyspnea12 Questionnaire $(\mathrm{p}<0.001, \mathrm{p}<0.001 ; \mathrm{p}=0.047, \mathrm{p}<0.001 ; \mathrm{p}<0.001, \mathrm{p}<0.00,1$, respectively).Patients who not receiving oxygen therapy had higher RCSQ mean scores and lower Dyspnea-12 Questionnaire scores $(\mathrm{p}<0.001, \mathrm{p}<0.001 ; \mathrm{p}<0.001, \mathrm{p}<0.001$, respectively). There was a strong negative relationship between the total scores obtained from the Richards-Campbell Sleep Questionnaire and the Dyspnea-12 Questionnaire $(\mathrm{r}=-701, \mathrm{p}<.001)$. Conclusions: Sleep quality is affected by dyspnea severity in patients with COVID-19. Sleep quality and dyspnea severity are also influenced by quite different factors, and these should be addressed and eliminated by nurses as part of a holistic approach. The results of this study will help nurses, especially those providing treatment and care for patients with COVID-19, to identify the factors affecting dyspnea and sleep quality and to plan, implement, and evaluate nursing interventions that will reduce their workload.
\end{abstract}

